International Journal of Pure and Applied Mathematics

Volume 95 No. $1 \quad 2014,23-29$

ISSN: 1311-8080 (printed version); ISSN: 1314-3395 (on-line version)

url: http://www.ijpam.eu

doi: http://dx.doi.org/10.12732/ijpam.v95i1.3

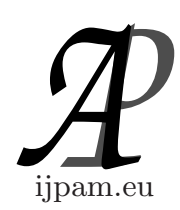

\title{
RN HYERS-ULAM-RASSIAS STABILITY OF FUNCTIONAL EQUATIONS: A DIRECT METHOD
}

\author{
D.Y. Shin ${ }^{1}$, H. Azadi Kenary ${ }^{2}$, N. Sahami ${ }^{3}$ \\ ${ }^{1}$ Department of Mathematics \\ College of Natural Science \\ University of Seoul \\ KOREA \\ ${ }^{2}$ Department of Mathematics \\ Beyza Branch \\ Islamic Azad University \\ Beyza, IRAN
}

\begin{abstract}
In this paper we prove Hyres-Ulam-Rassias stability of an additive functional equation in random normed spaces. The concept of Hyers-UlamRassias stability originated from Th.M. Rassias stability theorem that appeared in his paper: On the stability of the linear mapping in Banach spaces, Proc. Amer. Math. Soc., 72 (1978), 297-300.
\end{abstract}

AMS Subject Classification: 39B22, 39B52, 39B22, 39B82, 46S10

Key Words: random stability, functional equations

\section{Preliminaries}

Definition 1.1. A function $F: \mathbb{R} \rightarrow[0,1]$ is called a distribution function if it is nondecreasing and left-continuous, with $\sup _{t \in R} F(t)=1$ and $i n f_{t \in R} F(t)=$ 0 . The class of all distribution functions $F$ with $F(0)=0$ is denoted by $D_{+}$. For every $a \geq 0, H_{a}$ is the element of $D_{+}$defined by

Received: $\quad$ May 9, 2013

(c) 2014 Academic Publications, Ltd.

${ }^{\S}$ Correspondence author url: www.acadpubl.eu 


$$
H_{a}(t)=\left\{\begin{array}{ll}
0 & \text { if } t \leq a \\
1 & \text { if } t>a
\end{array} .\right.
$$

Definition 1.2. Let $X$ be a real vector space, $\Psi$ be a mapping from $X$ into $D_{+}$(for any $x \in X, \Psi(x)$ is denoted by $\Psi_{x}$ ) and $T$ be a $t$-norm. The triple $(X, \Psi, T)$ is called a random normed space (briefly $R N$-space) iff the following conditions are satisfied:

(i) $\Psi_{x}=H_{0}(t)$ iff $x=\theta$, the null vector;

(ii) $\Psi_{\alpha x}(t)=\Psi_{x}\left(\frac{t}{|\alpha|}\right)$ for all $\alpha \in \mathbb{R}, \alpha \neq 0$ and $x \in X$.

(iii) $\Psi_{x+y}(t+s) \geq T\left(\Psi_{x}(t), \Psi_{y}(s)\right)$, for all $x, y \in X$ and $t, s>0$.

Every normed space $(X,\|\|$.$) defines a random normed space \left(X, \Psi, T_{M}\right)$ where for every $t>0$,

$$
\Psi_{u}(t)=\frac{t}{t+\|u\|}
$$

and $T_{M}$ is the minimum $t$-norm. This space is called the induced random normed space.

If the $t$-norm $T$ is such that $\sup _{0<a<1} T(a, a)=1$, then every $R N$-space $(X, \Psi, T)$ is a metrizable linear topological space with the topology $\tau$ (called the $\Psi$-topology or the $(\epsilon, \delta)$-topology) induced by the base of neighborhoods of $\theta,\{U(\epsilon, \lambda) \mid \epsilon>0, \lambda \in(0,1)\}$, where

$$
U(\epsilon, \lambda)=\left\{x \in X \mid \Psi_{x}(\epsilon)>1-\lambda\right\}
$$

Definition 1.3. A sequence $\left\{x_{n}\right\}$ in an $R N$-space $(X, \Psi, T)$ converges to $x \in X$, in the topology $\tau$ (we denote $\lim x_{n}=x$ ) if $\lim _{n \rightarrow \infty} \Psi_{x_{n}-x}(t)=1$, $\forall t>0$.

Definition 1.4. A sequence $\left\{x_{n}\right\}$ is called Cauchy sequence if for all $t>0$, $\lim _{n \rightarrow \infty} \Psi_{x_{n}-x_{m}}(t)=1$. The $R N$-space $(X, \Psi, T)$ is said to be complete if every Cauchy sequence in $X$ is convergent.

\section{Random Stability of the Functional Equation$$
f(m x+n y)=m f(x)+n f(y)
$$

Throughout this section, using direct method we prove the Hyers-Ulam-Rassias stability of functional equation $f(m x+n y)=m f(x)+n f(y)$ in random normed $\operatorname{spaces}($ where $m, n \in \mathcal{N})$. 
Theorem 2.1. Let $X$ be a vector space, $(Z, \Psi, \min )$ be an $R N$-space, and $\psi: X^{m} \rightarrow Z$ be a function such that for some $0<\alpha<m+n$,

$$
\Psi_{\psi((m+n) x,(m+n) y)}(t) \geq \Psi_{\alpha \psi(x, y)}(t) . \quad \forall x, y \in X, t>0
$$

Also, for all $x, y \in X$ and $t>0$

$$
\lim _{n \rightarrow \infty} \Psi_{\psi\left((m+n)^{p} x,(m+n)^{p} y\right)}\left((m+n)^{p} t\right)=1 .
$$

If $(Y, \mu, \min )$ be a complete $R N$-space. If $f: X \rightarrow Y$ is a mapping such that for all $x, y \in X$ and $t>0$

$$
\mu_{f(m x+n y)-m f(x)-n f(y)}(t) \geq \Psi_{\psi(x, y)}(t),
$$

then there is a unique additive mapping $C(x): X \rightarrow Y$ such that

$$
\mu_{f(x)-C(x)}(t) \geq \Psi_{\psi(x, x)}((m+n-\alpha) t) .
$$

Proof. Putting $y=x$ in (4.2) we see that for all $x \in X$,

$$
\mu_{\frac{f((m+n) x)}{m+n}-f(x)}(t) \geq \Psi_{\psi(x, x)}((m+n) t) .
$$

Replacing $x$ by $(m+n)^{p} x$ in (4.4) and using (4.1), we obtain

$$
\begin{aligned}
\mu_{\frac{f\left((m+n) p^{p+1} x\right)}{(m+n)^{p+1}}-\frac{f\left((m+n) p_{x}\right)}{(m+n)^{p}}}(t) & \geq \Psi_{\psi\left((m+n)^{p} x,(m+n)^{p} x\right)}\left((m+n)^{p+1} t\right) \\
& \geq \Psi_{\psi(x, x)}\left(\frac{(m+n)^{p+1} t}{\alpha^{p}}\right) .
\end{aligned}
$$

So by (4.5) we obtain

$$
\begin{aligned}
& \mu_{\frac{f\left((m+n)^{p} x\right)}{(m+n)^{p}}-f(x)}\left(\sum_{k=0}^{p-1} \frac{t \alpha^{k}}{(m+n)^{k+1}}\right) \\
= & \mu_{\sum_{k=0}^{p-1} \frac{f\left((m+n)^{k+1} x\right)}{(m+n)^{k+1}}-\frac{f\left((m+n)^{k}\right)}{(m+n)^{k}}}\left(\sum_{k=0}^{p-1} \frac{t \alpha^{k}}{(m+n)^{k+1}}\right) \\
\geq & T_{k=0}^{p-1}\left(\mu_{\frac{f\left((m+n)^{k+1} x\right)}{(m+n)^{k+1}}-\frac{f\left((m+n)^{k} x\right)}{(m+n)^{k}}}\left(\frac{t \alpha^{k}}{(m+n)^{k+1}}\right)\right) \\
\geq & T_{k=0}^{p-1}\left(\Psi_{\psi(x, x)}(t)\right) \\
= & \Psi_{\psi(x, x)}(t) .
\end{aligned}
$$


This implies that

$$
\mu_{\frac{f\left((m+n)^{p}\right)}{(m+n)^{p}}-f(x)}(t) \geq \Psi_{\psi(x, x)}\left(\frac{t}{\sum_{k=0}^{p-1} \frac{\alpha^{k}}{(m+n)^{k+1}}}\right) .
$$

Replacing $x$ by $(m+n)^{q} x$ in (4.7), we obtain

$$
\begin{aligned}
\mu_{\frac{f\left((m+n)^{p+q} x\right)}{(m+n)^{p+q}}-\frac{f\left((m+n)^{q}\right)}{(m+n)^{q}}}(t) & \geq \Psi_{\psi\left((m+n)^{q} x,(m+n)^{q} x\right)}\left(\frac{t}{\sum_{k=0}^{p-1} \frac{\alpha^{k}}{(m+n)^{k+q+1}}}\right) \\
& \geq \Psi_{\psi(x, x)}\left(\frac{t}{\sum_{k=0}^{p-1} \frac{\alpha^{k+q}}{(m+n)^{k+q+1}}}\right) \\
& =\Psi_{\psi(x, x)}\left(\frac{t}{\sum_{k=q}^{p+q-1} \frac{\alpha^{k}}{(m+n)^{k+1}}}\right) .
\end{aligned}
$$

As

$$
\lim _{p, q \rightarrow \infty} \Psi_{\psi(x, x)}\left(\frac{t}{\sum_{k=q}^{p+q-1} \frac{\alpha^{k}}{(m+n)^{k+1}}}\right)=1,
$$

then $\left\{\frac{f\left((m+n)^{p} x\right)}{(m+n)^{p}}\right\}_{n=1}^{+\infty}$ is a Cauchy sequence in complete RN-space $(Y, \mu$, min $)$, so there exist some point $C(x) \in Y$ such that

$$
C(x)=\lim _{n \rightarrow \infty} \frac{f\left((m+n)^{p} x\right)}{(m+n)^{p}} .
$$

Fix $x \in X$ and put $q=0$ in (4.8). Then we obtain

$$
\mu_{\frac{f\left((m+n)^{p}\right)}{(m+n)^{p}}-f(x)}(t) \geq \Psi_{\psi(x, x)}\left(\frac{t}{\sum_{k=0}^{p-1} \frac{\alpha^{k}}{(m+n)^{k+1}}}\right) .
$$

and so, for every $\epsilon>0$, we have

$$
\begin{aligned}
\mu_{C(x)-f(x)}(t+\epsilon) & \geq T\left(\mu_{C(x)-\frac{f\left((m+n)^{p}\right)}{(m+n)^{p}}}(\epsilon), \mu_{\frac{f\left((m+n)^{p}\right)}{(m+n)^{p}}-f(x)}(t)\right) \\
& \geq T\left(\mu_{C(x)-\frac{f\left((m+n)^{p} x\right)}{(m+n)^{p}}}(\epsilon), \Psi_{\psi(x, x)}\left(\frac{t}{\sum_{k=0}^{p-1} \frac{\alpha^{k}}{(m+n)^{k+1}}}\right)\right) .
\end{aligned}
$$

Taking the limit as $p \rightarrow \infty$, we get

$$
\mu_{C(x)-f(x)}(t+\epsilon) \geq \Psi_{\psi(x, x)}((m+n-\alpha) t) .
$$

Since $\epsilon$ was arbitrary by taking $\epsilon \rightarrow 0$ in (4.10), we obtain

$$
\mu_{C(x)-f(x)}(t) \geq \Psi_{\psi(x, x)}((m+n-\alpha) t) .
$$


Replacing $x$ and $y$ by $(m+n)^{p} x$ and $(m+n)^{p} y$ respectively, in (4.2) and using this fact that $\lim _{p \rightarrow \infty} \Psi_{\psi\left((m+n)^{p} x,(m+n)^{p} y\right)}\left((m+n)^{p} t\right)=1$, we get for all $x, y \in X$ and for all $t>0$,

$$
C(m x+n y)=m C(x)+n C(y) .
$$

To prove the uniqueness of the additive mapping $C$, assume that there exist another additive mapping $D: X \rightarrow Y$ which satisfies (4.3). Since for all $p \in \mathbb{N}$ and every $x \in X$,

$$
C\left((m+n)^{p} x\right)=(m+n)^{p} C(x) \text { and } D\left((m+n)^{p} x\right)=(m+n)^{p} D(x),
$$

we find that

$$
\mu_{C(x)-D(x)}(t)=\lim _{n \rightarrow \infty} \mu_{\frac{C\left((m+n)^{p} x\right)}{(m+n)^{p}}-\frac{D\left((m+n)^{p}\right)}{(m+n)^{p}}}(t) .
$$

So

$$
\begin{aligned}
& \mu_{\frac{C\left((m+n) p_{x}\right)}{(m+n)^{p}}-\frac{D\left((m+n) p_{x}\right)}{(m+n)^{p}}(t)} \\
\geq & \min \left\{\mu_{\frac{C\left((m+n) p_{x}\right)}{(m+n)^{p}}-\frac{f\left((m+n)^{p}\right)}{(m+n)^{p}}}\left(\frac{t}{2}\right), \mu_{\frac{D\left((m+n)^{p}\right)}{(m+n)^{p}}-\frac{f\left((m+n) p_{x}\right)}{(m+n)^{p}}}\left(\frac{t}{2}\right)\right\} \\
\geq & \Psi_{\psi\left((m+n)^{p} x,(m+n)^{p} x\right)}\left(\frac{(m+n)^{p}(m+n-\alpha) t}{2}\right) \\
\geq & \Psi_{\psi(x, x)}\left(\frac{(m+n)^{p}(m+n-\alpha) t}{2 \alpha^{p}}\right) .
\end{aligned}
$$

Since $\lim _{p \rightarrow \infty} \frac{(m+n)^{p}(m+n-\alpha) t}{2 \alpha^{p}}=\infty$, we get

$$
\lim _{p \rightarrow \infty} \Psi_{\psi(x, x)} \frac{(m+n)^{p}(m+n-\alpha) t}{2 \alpha^{p}}=1 .
$$

Therefore, it follows that for all $t>0, \mu_{C(x)-D(x)}(t)=1$ and so $C(x)=D(x)$. This completes the proof.

Corollary 2.1. Let $X$ be a real linear space, $(Z, \Psi, \min )$ be an $R N$-space and $(Y, \mu$, min $)$ a complete $R N$-space. Let $p \in(0,1)$ and $z_{0} \in Z$. If $f: X \rightarrow Y$ is a mapping such that for all $x, y \in X$ and $t>0$

$$
\mu_{f(m x+n y)-m f(x)-n f(y)}(t) \geq \Psi_{\left.\|x\|\right|_{z_{0}}}(t),
$$

then there is a unique additive mapping $C(x): X \rightarrow Y$ such that

$$
\mu_{f(x)-C(x)}(t) \geq \Psi_{\|x\|^{p} z_{0}}\left(\left(m+n-(m+n)^{p}\right) t\right)
$$


Proof. Let $\alpha=(m+n)^{p}$ and $\psi: X^{2} \rightarrow Z$ be defined by $\psi(x, y)=\|x\|^{p} z_{0}$.

Corollary 2.2. Let $X$ be a real linear space, $(Z, \Psi$, min $)$ be an $R N$-space and $(Y, \mu$, min $)$ a complete $R N$-space. Let $p \in(0,1)$ and $z_{0} \in Z$. If $f: X \rightarrow Y$ is a mapping such that for all $x, y \in X$ and $t>0$

$$
\mu_{f(m x+n y)-m f(x)-n f(y)}(t) \geq \Psi_{\left(\|x\|^{p}+\|y\|^{p}\right) z_{0}}(t),
$$

then there is a unique additive mapping $C(x): X \rightarrow Y$ such that

$$
\mu_{f(x)-C(x)}(t) \geq \Psi_{\|x\|^{p}}\left(\frac{\left(m+n-(m+n)^{p}\right) t}{2}\right) .
$$

Proof. Let $\alpha=(m+n)^{p}$ and $\psi: X^{2} \rightarrow Z$ be defined by $\psi(x, y)=\left(\|x\|^{p}+\right.$ $\left.\|y\|^{p}\right) z_{0}$.

Corollary 2.3. Let $X$ be a real linear space, $(Z, \Psi$, min) be an $R N$-space and $(Y, \mu, \min )$ a complete $R N$-space. Let $p, q \in \mathbb{R}^{+}$where $p+q \in(0,1)$ and $z_{0} \in Z$. If $f: X \rightarrow Y$ is a mapping such that for all $x, y \in X$ and $t>0$

$$
\mu_{f(m x+n y)-m f(x)-n f(y)}(t) \geq \Psi_{\left(\left.\|x\|\right|^{p+q}+\|y\|^{p+q}+\|x\|^{p} \cdot\|y\|^{q}\right) z_{0}}(t),
$$

then there is a unique additive mapping $C: X \rightarrow Y$ such that

$$
\mu_{f(x)-C(x)}(t) \geq \Psi_{\|x\|^{p+q} z_{0}}\left(\frac{\left(m+n-(m+n)^{p+q}\right) t}{3}\right)
$$

Proof. Let $\alpha=(m+n)^{p+q}$ and $\psi: X^{2} \rightarrow Z$ be defined by $\psi(x, y)=$ $\left(\|x\|^{p+q}+\|y\|^{p+q}+\|x\|^{p} \cdot\|y\|^{q}\right) z_{0}$.

Corollary 2.4. Let $X$ be a real linear space, $(Z, \Psi$, min $)$ be an $R N$-space and $(Y, \mu, \min )$ a complete $R N$-space. Let $z_{0} \in Z$ and $f: X \rightarrow Y$ is a mapping such that for all $x, y \in X$ and $t>0$

$$
\mu_{f(m x+n y)-m f(x)-n f(y)}(t) \geq \Psi_{\delta z_{0}}(t),
$$

then there is a unique additive mapping $C: X \rightarrow Y$ such that for all $x \in X$ and $t>0$

$$
\mu_{f(x)-C(x)}(t) \geq \Psi_{\delta z_{0}}((m+n-1) t) .
$$

Proof. Let $\alpha=1$ and $\psi: X^{2} \rightarrow Z$ be defined by $\psi(x, y)=\delta z_{0}$.

\section{Acknowledgment:}

D.Y. Shin was supported by Basic Science Research Program through the National Research Foundation of Korea funded by the Ministry of Education, Science and Technology (NRF-2010-0021792). 


\section{References}

[1] S. Abbasbandy, T. Allahviranloo, Numerical solutions of fuzzy differential equations by taylor method, Computational Methods in Applied Mathematics 2 (2002) 113-124.

[2] T. Allahviranloo, N. Ahmady, E. Ahmady, Numerical solution of fuzzy differential equations by predictorcorrector method, Information Sciences 177 (2007) 1633-1647.

[3] D. Dubois, H. Prade, Towards fuzzy differential calculus, Fuzzy Sets and Systems 8 (1982) 1-7.

[4] S.S.L. Chang, L. Zadeh, On fuzzy mapping and control, IEEE Trans. Systems Man Cybernet. 2 (1972) 30-34.

[5] Lai Y. J., C. L. Hwang, Fuzzy Mathematical programming theory and applications, Springer, Belin, (1992). 
